\title{
Measurement of velocity field in parametrically excited solitary waves
}

\author{
Leonardo Gordillo ${ }^{1,2} \dagger$ and Nicolás Mujica ${ }^{1}$ \\ ${ }^{1}$ Departamento de Física, Facultad de Ciencias Físicas y Matemáticas, Universidad de Chile, \\ Casilla 487-3, Santiago, Chile \\ ${ }^{2}$ Laboratoire "Matière et Systèmes Complexes" (MSC), UMR 7057 CNRS, Université Paris 7 \\ Diderot, 75205 Paris Cedex 13, France
}

(Received ?; revised ?; accepted ?. - To be entered by editorial office)

Parametrically excited solitary waves emerge as localized structures in high-aspect-ratio free surfaces subject to vertical vibrations. Herein, we provide the first experimental characterization of the hydrodynamics of these waves using Particle Image Velocimetry. We show that the underlying velocity field of parametrically excited solitary waves is mainly composed by an oscillatory velocity field. Our results confirm the accuracy of Hamiltonian models with added dissipation in describing this field. Remarkably, our measurements also uncover the onset of a streaming velocity field which is shown to be as important as other crucial nonlinear terms in the current theory. The observed streaming pattern is particularly interesting due to the presence of oscillatory meniscii.

\section{Introduction}

Parametric instabilities in spatially extended systems can generate waves by their resonance with an external driving, which is a universal mechanism to generate structures in dissipative systems. In hydrodynamics, these structures satisfy a simple rule: energy losses due to viscous effects are compensated by the external injection of energy, e.g. by means of vertical vibrations. This balance can create extended or solitary structures that remain stable as long as the system is driven by the external force. In particular, solitary waves emerge in high-aspect-ratio free surfaces subject to vertical vibrations as a result of exciting the system at double the frequency of the first transverse mode. They become stable only after perturbing the free surface. Although these waves keep the sloshing motion features of the first transverse mode, their motion is highly localized in the longitudinal direction instead of involving the whole surface (Wu et al. 1984). This solitary wave is usually referred as non-propagating hydrodynamic soliton or parametrically excited solitary waves. The spatial envelopes are steady, very stable and do not propagate in contrast with classical hydrodynamic solitons. Remarkably, this dynamic behaviour can be found in several hydrodynamic systems: oscillons in Faraday configuration (Arbell \& Fineberg 2000) or solitary waves in vibrated Hele-Shaw cells (Rajchenbach et al. 2011) display spatial envelopes with similar spatiotemporal features.

Parametrically excited solitary waves are modelled in terms of the parametric dissipative nonlinear Schrödinger equation (pdNLSe), derived by Miles 1984b. This equation captures the minimum requirements for parametrically sustained one-dimensional solitary structures. Hence, its scope goes far beyond hydrodynamics (see e.g. Barashenkov et al. 1991; Denardo et al. 1992). In this regard, recent studies have been focused on

$\dagger$ Email address for correspondence: leonardo.gordillo@univ-paris-diderot.fr 
providing an exhaustive analysis of the pdNLSe using mathematical and numerical techniques. Experiments have also been used for this purpose (see Zhang et al. 2007; Gordillo et al. 2011; Clerc et al. 2011). On the contrary, some fundamental issues such as the validity of the approximations that yield the pdNLSe have remained unaddressed, with rare systematic comparisons between predictions and measurements (Chen et al. 1999). For worse, all the experimental characterizations that can be found in the literature have been achieved using a single technique, i.e. by tracking the free surface, a measurement useful for outlining solitary-waves stability and interaction laws but blind to potential underlying phenomena. This is a critical issue, since most steps involved in the pdNLSe derivation lie on strong hypotheses from fluid dynamics. Uncovering the velocity field beneath the structures is hence fundamental for a comprehensive experimental analysis of parametrically excited solitary waves.

In this article, we present experimental results concerning parametrically excited solitary waves with a close view on the hydrodynamics. The velocity fields that support the localized cross waves have been measured using Particle Image Velocimetry (PIV). The article is organized as follows. In $\S 2$, we outline the pdNLSe theoretical model. In §3, we describe briefly the experimental setup. A summary of our most significant experimental results can be found in $\S 4$. Finally, discussion and conclusions, including comparisons with pdNLSe theoretical predictions, are given in $§ 5$.

\section{Theory}

Consider a fluid layer of depth $d$ in an infinite channel of breadth $b$. The channel is oriented in the $x$ axis (walls at $y= \pm \frac{1}{2} b$ and $z=-d$ ) and forced to oscillate vertically at frequency $2 \omega$ and acceleration amplitude $\Gamma_{0}$. The vertical acceleration of the channel is accordingly $\Gamma(t)=-\Gamma_{0} \cos 2 \omega t$. Let us assume that $\omega$ approaches $\omega_{01}$, the first-transverse-mode frequency so the $(0,1)$ mode is parametrically excited. The linear theory of gravity waves for inviscid flows provides a good estimation for the $(0,1)$-mode frequency, $\omega_{01}=\sqrt{g k \tau}$, where $g$ is the acceleration gravity, $k=\pi / b$ is the wavenumber and $\tau \equiv \tanh k d$. The parametric forcing can thus be characterized in terms of two dimensionless parameters: the detuning $\nu=\frac{1}{2}\left(\omega^{2} / \omega_{01}^{2}-1\right)$ and the normalized acceleration amplitude $\gamma=\frac{1}{4} \Gamma_{0} / g$.

Free-surface waves on constant-depth inviscid flows are known to satisfy Hamiltonian properties (cf. Miles 1977). This can be used as a point of departure for deriving amplitude equations in such kind of system. However, realistic setups cannot be considered as conservative: external forcing is required to create structures. In any case, although viscous effect are neglected a priori in Hamiltonian formulations, adding linear dissipation in amplitude equations seems to be enough for modelling slightly viscous flows (Miles 1976). The reason is simple: in this kind of flow, the motion is basically inviscid all over the space except in thin boundary layers. Energy is thus dissipated without affecting the general features of the waves (cf. Miles 1967). For instance, the decay rate for the $(0,1)$ mode, $\alpha_{01}$, can be estimated from boundary layer analysis (Miles 1984b). In our problem, this provides an extra dimensionless parameter, the damping rate $\mu=\alpha_{01} / \omega_{01}$.

Using the stated hypotheses, it can be shown rigorously that the first transverse mode in an infinite channel can be modelled with (Miles 1984b)

$$
\mathrm{i}\left(\partial_{T} \psi+\mu \psi\right)=\nu \psi+2|\psi|^{2} \psi+\partial_{X X} \psi+\gamma \bar{\psi},
$$

which is the parametric dissipative nonlinear Schrödinger equation (pdNLSe). Notice that this equation is written in terms of the dimensionless variables $T=\omega_{01} t$ and $X=b^{-1 / 2} k x$, where $t$ stands for time, $x$ for the longitudinal spatial coordinate and 
$b=\frac{1}{4}\left[1+k d\left(1-\tau^{2}\right) / \tau\right]$. The complex field $\psi(X, T)$ contains the slow spatiotemporal modulation of the first transverse mode. In general, the deformation at the free surface $\eta(x, y, t)$ and the velocity potential inside the fluid, $\Phi(x, y, z, T)$, are related to $\psi(X, T)$ by means of

$$
\begin{aligned}
\eta(x, y, t) & =\operatorname{Re}\{a \psi(X, T) \exp i \omega t\} \sin k y, \\
\Phi(x, y, z, t) & =\operatorname{Im}\{a \psi(X, T) \exp i \omega t\} \frac{g \sin k y \cosh k(z+d)}{\omega_{01} \cosh k d},
\end{aligned}
$$

where $a^{2}=128 k^{-2} /\left(6 \tau^{2}-5+16 \tau^{-2}-9 \tau^{-4}\right)$.

Just as their propagating counterparts, non-propagating hydrodynamic solitons arise from a subtle balance between linearities, nonlinearities and dispersion. Parametrically excited solitary waves can be found by assuming solutions of the form $\psi(X, T)=$ $\rho(X) e^{-\mathrm{i} \theta}$. Straightforward calculations show the onset of a subcritical instability for $\gamma>\mu$ and $\nu<0$ with two families of solutions. One family of solutions is always unstable whereas the other one,

$$
\psi(X, T)= \pm \mathrm{i} \delta \operatorname{sech}\left[\delta\left(X-X_{0}\right)\right] e^{\frac{i}{2} \sin ^{-1} \frac{\mu}{\gamma}}
$$

is stable whenever $\gamma^{2}<\nu^{2}+\mu^{2}$; provided that $\delta^{2}=-\nu+\left(\gamma^{2}-\mu^{2}\right)^{\frac{1}{2}}$ (see Laedke $\&$ Spatschek 1991). The free parameter $x_{0}$ comes out from a constant of integration and stands for the position of the envelope centre of mass. Besides, (2.4) consists of two solutions with opposite sign. This is consistent with experimental observations of a soliton sort of polarity, a crucial feature for pair interactions (Wang \& Wei 1994; Wang et al. 1996; Clerc et al. 2009). Equation 2.1 also supports cnoidal and dnoidal families of solutions (cf. Miles 1984b; Umeki 1991). Besides, a change of sign in its nonlinear term gives rise to the kink-type solutions observed by Denardo et al. 1990. This is achieved for instance by decreasing the depth of the fluid layer (see also Miles 1984b, pp. 455-456).

\section{Experimental setup}

We run our experiments in an acrylic basin attached to an electromagnetic shaker (see figure 1). The trough of length $l=19.05 \mathrm{~cm}$ and breadth $b=2.54 \mathrm{~cm}$ was filled with an aqueous solution to a depth $d=2.00 \mathrm{~cm}$. The aqueous solution contained $2 \mathrm{ml}$ of Photoflo, used for improving wall wetting (Wu et al. 1984), and some $\mathrm{KBr}$ (13\% in mass concentration) for increasing the fluid density. The solution density matches that of the PIV particles, $1.1 \mathrm{~g} \cdot \mathrm{cm}^{-3}$, so particle settling becomes noticeable only after several hours (kinematic viscosity is also reduced in about 15\%). The acceleration of the basin was registered using a piezoelectric accelerometer and a lock-in amplifier, which was referenced externally to the input shaker signal. Parametrically excited solitary waves can be observed when the system is accelerated vertically as $\Gamma(t)=-\Gamma_{0} \cos (2 \pi f t)$, at frequencies $f$ slightly below $11 \mathrm{~Hz}$ and acceleration amplitudes $\Gamma_{0}$ around $0.1 g$. The frequency threshold is very close to the the double of the experimental first transversemode frequency, $f_{0,1}=5.49 \mathrm{~Hz}$, which was obtained by measuring the linear surface response using a capacitive sensor and a spectrum analyser in frequency-sweep mode (for more details, cf. Gordillo (2012)).

The PIV particles inside the fluid (carboxy-modified acrylate resin, $\varnothing=15 \mu \mathrm{m}$ ) were illuminated using a double-pulsed Nd:YAG laser (70 mJ per pulse) and a laser sheet generator. We placed the latter on a linear translational stage so the sheet position along the fluid layer $y$ could be easily adjusted. The laser sheet thickness inside the fluid was $2 \mathrm{~mm}$. Notice that illumination from the bottom is the only one compatible with PIV 


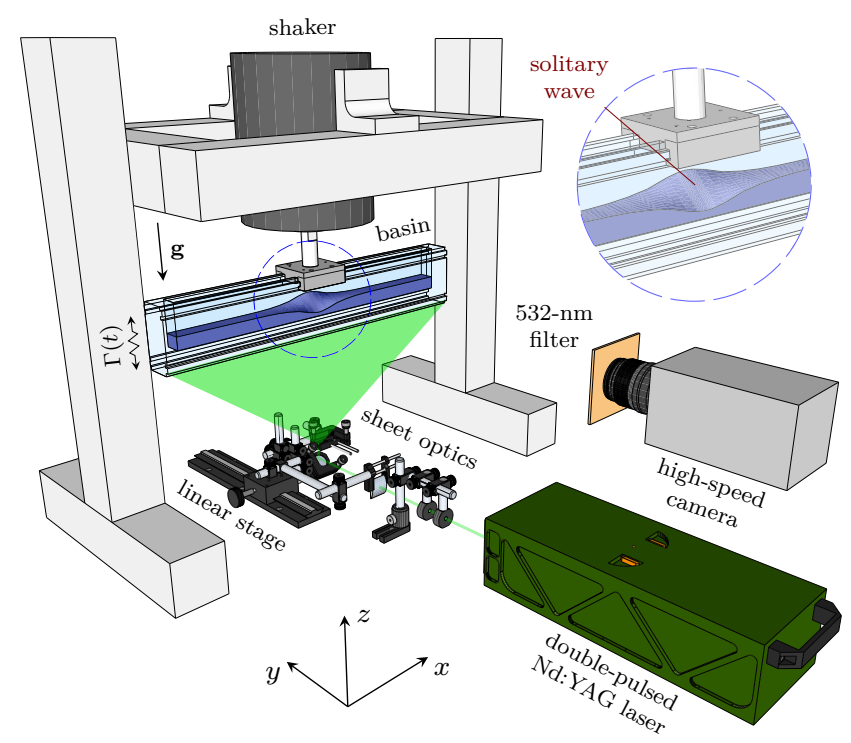

FiguRE 1. General scheme of the setup. Solitary waves are created in the fluid contained by a basin subject to vertical vibrations. The fluid is seeded with fluorescent particles and illuminated by a vertical laser sheet shiftable in the $y$ direction. PIV digital processing provides the velocity field in the $x-z$ plane at fixed $y_{s}$.

and measurement requirements. Unfortunately, due to the back and forth sloshing of the solitary waves (see zoom window in figure 1), the free surface reflects a huge amount of light on the $y$ direction. To avoid this, we used fluorescent PIV particles (absorption and emission peaks at 550 and $580 \mathrm{~nm}$ respectively) and blocked reflections with a longpass optical filter whose cut-off matches the light-source wavelength $(532 \mathrm{~nm})$.

Images were acquired using a high-speed camera providing an imaging region of $2560 \times$ 512 pixels $\left(20.0 \times 4.0 \mathrm{~cm}^{2}\right)$. We synchronized the laser double pulses $(\Delta t=10 \mathrm{~ms})$ with the motion of the solitary wave at some fixed phase $\theta_{s}$. Since the solitary wave sloshes at $\frac{1}{2} f$ and at a fixed phase with respect to the shaker input signal, the latter signal was used as the reference. The frames were acquired synchronously with the laser pulses. Each run consisted of 200 images pairs for fixed sheet position $y_{s}$ and solitary-wave phase $\theta_{s}$. A whole set of 36 different $\theta_{s}$ values were analysed throughout the whole solitary-wave cycle. Besides, 10 laser sheet positions $y_{s}$ across the fluid layer were also analysed for a fixed phase, $\theta_{s}=\pi$, at which the free-surface deformation is zero and the velocity is maximal on the front wall.

Image processing was performed using our own Matlab code with classical PIV digital techniques. Since image sequences included a moving boundary, we required an automatic algorithm for boundary detection. We achieved this by using a Radon-transformbased method from Sanchis \& Jensen (2011) on averaged same-phase samples. Likewise, background and illumination issues were corrected using statistics-based images. The calculated boundaries were then used for creating binary masks, which in turn were used in a multi-pass interrogation PIV scheme. The minimum interrogation-window size was $16 \times 16$ pixels $\left(1.25 \times 1.25 \mathrm{~mm}^{2}\right)$. The results presented here were averaged over the 200 samples in the correlation-function space to improve signal-to-noise ratio. 


\section{Results}

As a consequence of pulsed-laser synchronization, image sequences are composed of a series of double fast snapshots $(\Delta t=10 \mathrm{~ms})$ captured every soliton period $\left(\Delta t^{\prime}=2 / f\right)$. This temporal scheme provides sets of time-resolved velocity fields for a fixed phase between the external forcing and the soliton oscillation. Tuning this phase, the velocity field during the whole cycle can be found. The resulting velocity field $(u, v, w)$ is mainly oscillatory in time, similar to those of stationary waves. But the temporal scheme provides more than that. A simple inspection of sequences shows that after one cycle, seeding particles do not return to their position at the preceding cycle. If one frame is skipped such that the temporal scheme is stroboscopic, it is easy to notice that particles are constantly streamed. The effect of this streaming velocity field $(\bar{u}, \bar{v}, \bar{w})$ becomes perceptible in particle trajectories only after one or several cycles. In this sense, the instantaneous velocity field $(u, v, w)$ can be considered as the sum of two components: the oscillatory part, $(u-\bar{u}, v-\bar{v}, w-\bar{w})$, and the streaming one, $(\bar{u}, \bar{v}, \bar{w})$.

In figure 2 , we depict the velocity field inside the bulk of parametrically excited solitary wave at the phase $\theta_{s}=\pi$. Figures $2(a, b)$ display respectively the instantaneous and the streaming velocity fields in the $x-z$ plane for a fixed $y_{s}=-1.07 \mathrm{~cm}$. The driving frequency and amplitude for the displayed solitary waves were $f=10.9 \mathrm{~Hz}$ and $\Gamma_{0}=0.096 \mathrm{~g}$. The solitary-wave envelope is centred in the basin $\left(x_{0}=0\right.$ in equation 2.4). Corresponding videos can be viewed as supplementary material available online at: insert link.

In figure 3 , we include also side views ( $y$ - $z$ plane) of the velocity field captured at the same phase $\theta_{s}=\pi$. For this sequence, the basin was rotated $90^{\circ}$ in the $x-y$ plane (the axes remain fixed to the basin). To obtain a full view of the velocity field of this plane, we used a half solitary wave pinned at $x_{0}=\frac{1}{2} l$ instead of a centred one. In this configuration and due to the refraction of rays of light on the free surface, the uppermost region of a solitary wave centred at $x_{0}=0$ cannot be observed in images. Recalling that half solitary waves exist in a particular region of parameters, the frequency and amplitude of the external driving were suitably adjusted to $f=10.97 \mathrm{~Hz}$ and $\Gamma_{0}=0.127 g$. Figures $3(a, b)$ display the instantaneous and the streaming velocity fields. For both fields, the position of the laser sheet was $x_{s}=9.23 \mathrm{~cm}$. On this side view, the zoomed images $(1280 \times 1600$ pixels in a $2.8 \times 3.5 \mathrm{~cm}^{2}$ window; minimum interrogation window: $16 \times 16$ pixels, i.e. $0.35 \times 0.35 \mathrm{~mm}^{2}$ ) allows to resolve the meniscus formed at the front and back walls. In the figure, the white background represents image regions occupied by the fluid. More videos are available online at: insert link.

\subsection{Instantaneous velocity field}

The instantaneous velocity field inside the bulk of the parametrically excited solitary wave is mainly given by an oscillatory part. Before analysing any data, the uniform velocity due to the driving of the basin was subtracted so the velocity fields are in a frame of reference fixed to the basin. The front view of the velocity field shows that the motion is highly localized in the envelope of the structure with magnitudes decreasing one order of magnitude from the centre to the side walls of the trough. The magnitude of the velocity increases also as we approach the free surface, which is a general feature of gravity waves in uniform depth containers. These two spatial features can be observed through all the cycle of the solitary wave: the direction of the arrows are mainly the same and only their magnitudes oscillate in time. Thus, a single-phase snapshot, e.g. figure $2(a)$, provides a good overview of the distribution of velocity in the $x-y$ plane. Besides, a fast inspection of the instantaneous velocity field at other planes by means of moving the laser sheet, shows that the spatial and temporal features in the $x$ - $z$ plane are the 

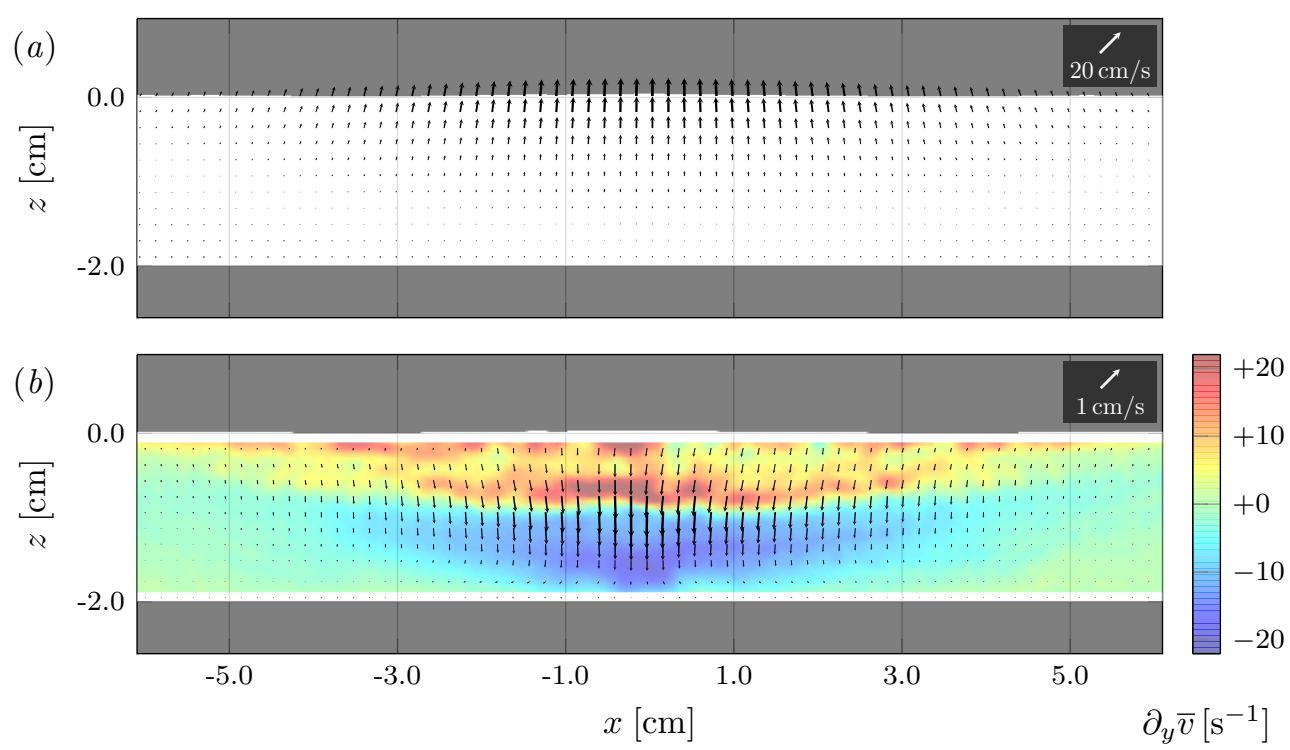

FigURE 2. Front view ( $x-y$ plane) of the velocity field of solitary wave whose envelope is centred at $x=0$ and $\theta_{s}=\pi$. Only the central region of the trough is shown. The laser sheet is placed at $y_{s}=1.07 \mathrm{~cm}$. (a) Instantaneous velocity field. (b) Streaming velocity field and out-of-plane velocity gradient $\partial_{y} \bar{v}$ (in colours). The arrow scales for each figure are also displayed.

$(a)$

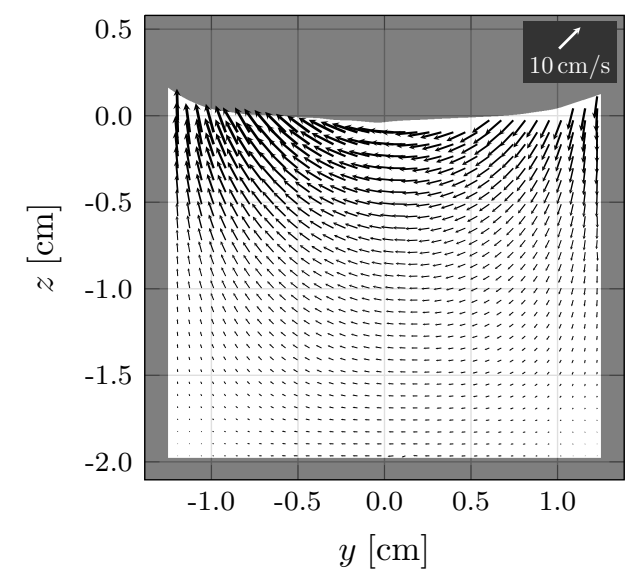

(b)

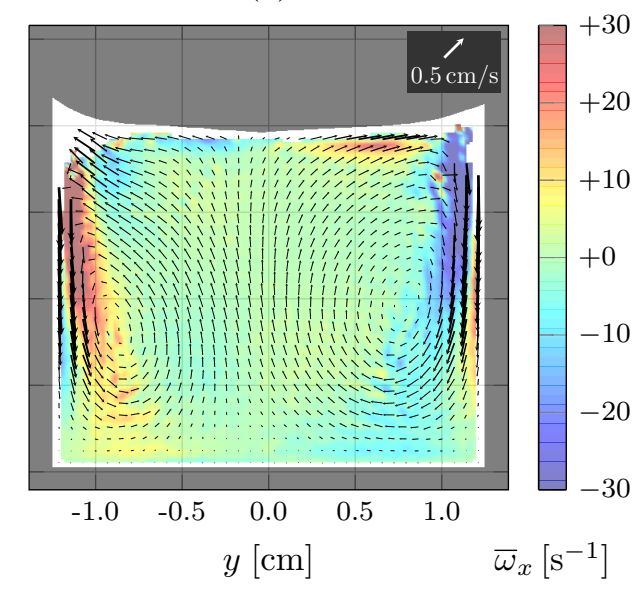

Figure 3. Front view ( $x-z$ plane) of the velocity field of half solitary wave pinned at a lateral wall at $\theta_{s}=\pi$. The laser sheet was placed at $x_{s}=9.23 \mathrm{~mm}$. (a) Instantaneous velocity field. $(b)$ Streaming velocity field and out-of-plane vorticity $\bar{\omega}_{x}$ (in colours).

same. The module of arrows just reduces as $y_{s}$ approaches the centre of the basin in the $y$ direction.

The last feature is in agreement with the velocity fields obtained from side views of the trough ( $y-z$ plane) as depicted in figure 3(a). The temporal features of this view match those from the $x-z$ plane: a field with static orientation and time-oscillating magnitudes. The snapshots also display that particles move from the positive side of the $y$ 
axis to the negative one as the free surface acquires its characteristic sloshing motion. The magnitudes increase when approaching the free surface as expected.

\subsection{Streaming flow}

In contrast to the instantaneous velocity field, the streaming flow does not oscillate during a cycle. By changing the phase of light pulses with respect to the solitary-wave cycle, we observed that the field is mainly steady across the bulk of the fluid. An example of the velocity field in the $x-z$ is shown in figure $2(b)$. The phase and $y_{s}$ position of the illuminated plane is the same as for figure $2(a)$. Notice that compared to the maximal instantaneous velocity, the magnitudes are around 20 times smaller. At this phase, we can observe that the particles are pushed downward and outward the solitary-wave core. Higher magnitudes are observed in the centre of the channel rather than close to the free surface. The distribution of the velocity field suggests an important out-of-plane velocity gradient(see colours in figure $2 b$ ). Particles are streamed into the plane at the top and out of the plane at the bottom. The opposite occurs at the centre of the basin $\left(y_{s}=0\right)$, where particles move upward everywhere.

To clarify the general structure of the streaming motion of particles, the $y-z$ view is very useful. The velocity field in figure $3(b)$ shows a pair of vortex-like structures aligned to the $x$ axis. As observed in the $x-z$ view, particles are streamed downward in the front and back walls $\left(y= \pm \frac{1}{2} b\right)$ and upward in the centre of the trough. Streaming near both menisci is hard to resolve since particle images are subject to high shear in this region. The vorticity distribution (shown in colours in figure $3 b$ ) is highly localized near the front and back walls. The vorticity core is pinched to the meniscus and slightly pushed back by the walls as $z$ decreases.

\section{Discussion and conclusions}

\subsection{Comparison with predicted results}

In general terms, the model of Miles (1984b) based on Hamiltonian equations and linear dissipation predicts well the deformation of the free surface of parametrically excited solitary waves (see Clerc et al. 2009; Gordillo et al. 2011). Herein, we display comparisons that now comprehend the velocity field inside the bulk of solitary waves. According to Miles theory, the velocity of the fluid underneath the free surface is irrotational and satisfies $(u, v, w)=\nabla \Phi$. The potential is given by

$$
\Phi(x, y, z, t)=\frac{ \pm a \delta g \sin k y \cosh k(z+d) \cos \theta_{s}(t)}{\omega_{01} \cosh k d \cosh \left[\delta b^{-1 / 2} k\left(x-x_{0}\right)\right]}
$$

where $\theta_{s}(t)=\omega t+\frac{1}{2} \sin ^{-1} \frac{\mu}{\gamma}$. Equation (5.1) can be used to derive the predicted components of the velocity. A simple way to test the accuracy of the model is to fit velocity-field projections from model (5.1) to the experimental data. To reduce the high dimensionality of the set of dependant variables (three for space and one for time), we fixed two dependant variables and applied a surface fit using the cftool function in Matlab on the remaining two.

In figure 4(a), we display the results for the oscillatory vertical velocity $(w-\bar{w})$ in the $x$ - $z$ plane. Here, $y$ is fixed at $y_{s}=1.07 \mathrm{~cm}$ and $t$ correspond to the two phases at which the velocities are maximal and minimal, i.e. $\theta_{s}=\{0, \pi\}$. For visualization, we collapsed the PIV experimental data for eight different $z$ values $(-1.5<z<0.5 \mathrm{~cm})$ into two master curves. This was done by dividing the data by the $z$-dependant part of $\partial_{z} \Phi$, i.e. $\sinh \left[K_{z}(z+d)\right]$. The curves should then be represented by a function $f(x)=$ $A \operatorname{sech} K_{x}\left(x-X_{0}\right)$. Variables in capital letters are fitted parameters. 
(a)

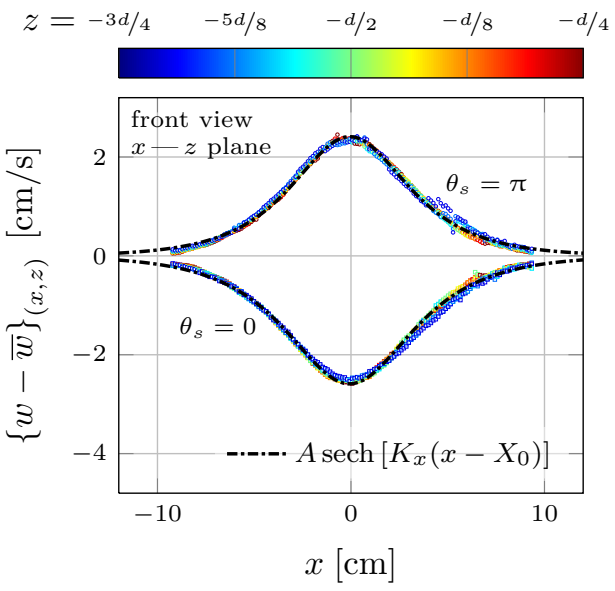

(b)

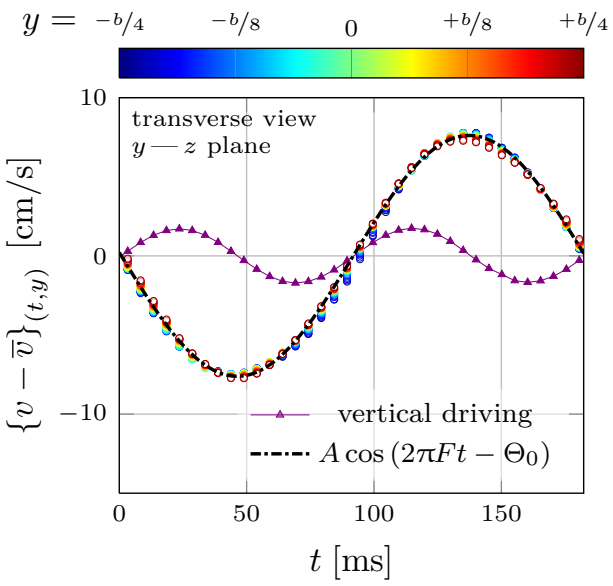

FIGURE 4. Surface fitting results of the potential model (5.1) for solitary waves. For visualization, surfaces are collapsed into single master curves (symbols) that are then compared to fitted model (dash-dotted lines). (a) Maximal and minimal oscillatory vertical velocity $w-\bar{w}$ in the $(x, z)$ plane. Fitting parameters: (upper curve) $A=2.41 \pm 0.04 \mathrm{~cm} / \mathrm{s}, K_{x}=0.373 \pm 0.002 \mathrm{~cm}^{-1}$, $K_{z}=1.36 \pm 0.01 \mathrm{~cm}^{-1}, X_{0}=-0.06 \pm 0.01 \mathrm{~cm}$; (lower curve) $A=-2.59 \pm 0.04 \mathrm{~cm} / \mathrm{s}$, $K_{x}=0.344 \pm 0.001 \mathrm{~cm}^{-1}, K_{z}=1.24 \pm 0.01 \mathrm{~cm}^{-1}, X_{0}=-0.08 \pm 0.01 \mathrm{~cm}$, (b) Oscillatory horizontal velocity $v-\bar{v}$ in the $(y, t)$ plane. Fitting parameters: $A=7.61 \pm 0.02 \mathrm{~cm} / \mathrm{s}$, $f=5.492 \pm 0.005 \mathrm{~Hz}, K_{y}=1.21 \pm 0.01 \mathrm{~cm}^{-1}, \Phi_{0}=-1.543 \pm 0.004$. The basin vertical velocity is also displayed.

A similar comparison for the temporal evolution of the velocity field of a half parametrically excited solitary wave is displayed in figure $4(b)$. In this case, we analysed the oscillatory horizontal velocity from the side views, $(v-\bar{v})$, in terms of the time $t$ and the horizontal coordinate $y$ at a fixed depth $z=-0.44 \mathrm{~cm}$ (the $x$ position is again fixed at $\left.x_{s}=9.23 \mathrm{~mm}\right)$. For visualization, the surface in the $(y, t)$ space was collapsed into a single master curve by diving the velocity profiles by the $y$-dependence part $\partial_{y} \Phi$, i.e. $\cos \left[K_{y} y\right]$. A set of 80 curves in the range $-0.64<y<0.64 \mathrm{~cm}$ were used for this purpose. According to (5.1), the velocity should be well fitted by $f(x)=A \cos \left(2 \pi F t-\Theta_{0}\right)$. The vertical velocity of the basin is also plotted displaying the parametric nature of the instability.

Figures $4(a-b)$ are strong proofs that the potential-velocity model describes well the oscillatory part of the velocity field. The hyperbolic secant profiles reproduce with excellent accuracy the velocity distribution along the solitary wave. The fitted values for maximal and minimal vertical velocity $(w-\bar{w})$ of $4(a)$ show good agreement between them. In general, the fitted values for $K_{y}$ and $K_{z}$ match well the crosswise wavenumber $k=\pi / b=1.24 \mathrm{~cm}^{-1}$. Accordingly, experimental measurements for the half solitary wave show a crosswise standing wave profile that oscillates harmonically at the double of the driving period.

\subsection{Parametric streaming}

At this point, Miles' model seems to be very accurate to reproduce experimental data. At least, when comparisons with experimental data are made after subtracting the mean streaming velocity field to the instantaneous one. This important step of processing is vital for the good agreement displayed in figures $4(a-b)$. Although Miles' model for Hamiltonian flows can yield some sort of streaming for higher-order terms (see Gordillo 
2012), these corrections remain potential across the whole bulk of the fluid. Hence, the model is blind to streaming flows with a vorticity distribution as the one that parametrically excited solitary waves support. Even more striking is the fact that the streaming flow is so significant. Using equation (2.3) and the formulas for the pdNLSe parameters, we can estimate roughly the following values from experimental data: $\psi \sim 10^{-1}$ and $\nu \sim \mu \sim \gamma \sim 10^{-1}$. Meanwhile, the ratio between the streaming and the potential velocities is $\bar{w} / w \approx 10^{-1}$. This means that corrections due to the coupling of streaming and potential-flow are $\psi^{3}$ and hence, as important as the the higher-order term in (2.1), which is actually responsible for the highly localized envelope of parametrically excited solitary waves. Two question naturally arises: What is the physical origin of the parametric streaming? Why Miles model and pdNLSe have been so successful in describing parametrically excited solitary waves despite they do not consider the streaming observed in experiments?

The physical origin of streaming in parametric flows has been widely studied in a domain slightly different: acoustic flows. Streaming occurs because oscillatory boundary layers transfer vorticity to the bulk of the fluid (see Batchelor, G. K. 2000, pp. 358-361 and references therein). The induced streaming velocity field is independent of the viscosity of the fluid $\mu_{0}$ and does not vanish as $\mu_{0} \rightarrow 0$ as a consequence of the singular limit of the Navier-Stokes equation in the high Reynolds number limit. Streaming in parametric instabilities were first visualized by Douady (1990) using Kalliroscope particles in a Faraday-instability configuration. To our knowledge, quantitative measurements of the streaming field in a parametric instability have never been reported. Theoretical analysis on this subject are also rare and have addressed to the bottom and free-surface boundary layers (Martín et al. 2002; Martín \& Vega 2005). Since parametrically excited solitary waves are necessarily supported between two vertical walls, we are sceptical about their applicability to our setup. Furthermore, it is clear that the effect of the advancing and receding menisci cannot be disregarded as they are crucial for the correct characterization of the vorticity field (see figure $3 b$ ).

Concerning the second question, the few theoretical works made on this subject can give us important clues. Martín et al. (2002) considered the problem of finding an amplitude equation for two-dimensional Faraday waves starting from Navier-Stokes equation in a laterally unbounded fluid. The resulting amplitude equation is similar to that obtained from Hamiltonian formulations Miles (1984a) except for an integral term that accounts for the coupling between streaming and the Faraday waves. This term does not generate a major change in the general dynamics of the amplitude equation although its vital to explain drift instability in Faraday waves (Martín et al. 2002). For parametrically excited solitary waves, although calculations are considerably more complex, streaming coupling should provide corrections to the $a$ factor, defined after equations (2.2) and (2.3), without modifying the dynamics of the pdNLSe equation. However, it is hard to establish the scope of this coupling term for further bifurcations. In order to accomplish the challenge of incorporating parametric streaming in the amplitude equation for parametrically excited solitary waves, we should undoubtedly address to a more fundamental hydrodynamical problem: understand and be able to predict streaming near oscillatory contact lines.

\section{Acknowledgements}

We are thankful to Edgar Knobloch and Marcel Clerc for fruitful discussions. The research was supported by Conicyt grants ACT 127 and AIC 43. LG acknowledges Conicyt fellowships 57080094 and 24100131, and the AXA Research Fund. 


\section{Supplementary movies}

Supplementary movies are available at ...

\section{REFERENCES}

Arbell, H. \& Fineberg, J. 2000 Temporally Harmonic Oscillons in Newtonian Fluids. Phys. Rev. Lett. 85 (4), 756-759.

Barashenkov, I. V., Bogdan, M. M. \& Korobov, V. I. 1991 Stability diagram of the phase-locked solitons in the parametrically driven, damped nonlinear Schrödinger equation. Europhys. Lett. 15, 113.

Batchelor, G. K. 2000 An Introduction to Fluid Dynamics. Cambridge, UK: Cambridge University Press.

Chen, Weizhong, Tu, Juan \& Wei, Rongue 1999 Onset instability to nonpropagating hydrodynamic solitons. Phys. Lett. A 255 (4), 272-276.

Clerc, M. G, Coulibaly, S., Gordillo, L, Mujica, N. \& Navarro, R. 2011 Coalescence cascade of dissipative solitons in parametrically driven systems. Phys. Rev. E 84 (3), 036205.

Clerc, M. G, Coulibaly, S., Mujica, N., Navarro, R. \& Sauma, T. 2009 Soliton pair interaction law in parametrically driven Newtonian fluid. Philos. T. Roy. Soc. A 367, 3213-3226.

Denardo, B., Galvin, B., Greenfield, A., Larraza, A., Putterman, S. J \& Wright, W. B 1992 Observations of localized structures in nonlinear lattices: Domain walls and kinks. Phys. Rev. Lett. 68 (1), 1730-1733.

Denardo, B., Wright, W. B, Putterman, S. J \& Larraza, A. 1990 Observation of a kink soliton on the surface of a liquid. Phys. Rev. Lett. 64 (1), 1518-1521.

Douady, S. 1990 Experimental study of the Faraday instability. J. Fluids Mech. 221, 383-409.

Gordillo, L 2012 Non-Propagating Hydrodynamic Solitons in a Quasi-One-Dimensional Free Surface Subject to Vertical Vibrations. PhD thesis, Universidad de Chile, Santiago.

Gordillo, L, Sauma, T., Zárate, Y., Espinoza, I., Clerc, M. G \& Mujica, N. 2011 Can non-propagating hydrodynamic solitons be forced to move? Eur. Phys. J. D 62 (1), 39-49.

Laedke, E. W. \& SpatscheK, K. H. 1991 On localized solutions in nonlinear Faraday resonance. J. Fluids Mech. 223, 589-601.

Martín, E., Martel, C. \& Vega, J. M 2002 Drift instability of standing Faraday waves. J. Fluids Mech. 467, 57-79.

MARTín, E. \& VEGA, J. M 2005 The effect of surface contamination on the drift instability of standing Faraday waves. J. Fluids Mech. 546, 203-225.

Miles, J. W. 1967 Surface-Wave Damping in Closed Basins. Proc. Roy. Soc. Lond. A 297, 459.

Miles, J. W. 1976 Nonlinear surface waves in closed basins. J. Fluids Mech. 75, 419-448.

Miles, J. W. 1977 On Hamilton's principle for surface waves. J. Fluids Mech. 83, 153-158.

Miles, J. W. 1984a Nonlinear Faraday resonance. J. Fluids Mech. 146, 285-302.

Miles, J. W. $1984 b$ Parametrically excited solitary waves. J. Fluids Mech. 148, 451-460.

Rajchenbach, J., Leroux, A. \& Clamond, D. 2011 New Standing Solitary Waves in Water. Phys. Rev. Lett. 107 (2), 024502.

SAnchis, A. \& Jensen, A. 2011 Dynamic masking of PIV images using the Radon transform in free surface flows. Exp. Fluids 51 (4), 871-880.

Umeki, M. 1991 Parametric Dissipative Nonlinear Schrödinger Equation. J. Phys. Soc. Jpn. $60(1), 146$.

Wang, Wei, Wang, Xinlong, Wang, J. \& Wei, Ronguue 1996 Dynamical behavior of parametrically excited solitary waves in Faraday's water trough experiment. Phys. Lett. A 219, $74-78$.

Wang, Xinlong \& Wei, Rongue 1994 Observations of collision behavior of parametrically excited standing solitons. Phys. Lett. A 192 (1), 1-4.

Wu, JunRu, KeOlian, R. \& Rudnick, I. 1984 Observation of a Nonpropagating Hydrodynamic Soliton. Phys. Rev. Lett. 52 (1), 1421-1424.

Zhang, Likun, Wang, Xinlong \& TAO, Zhiyong 2007 Spatiotemporal bifurcations of a parametrically excited solitary wave. Phys. Rev. E $\mathbf{7 5}$ (3). 